\title{
Spectrum-Aware Wireless Sensor Networks
}

\author{
Peng Du \\ Dept. of Computer Science and Information Systems \\ Birkbeck, University of London \\ Malet Street, London WC1E 7HX \\ Email: peng@dcs.bbk.ac.uk
}

\author{
Prof. George Roussos \\ Dept. of Computer Science and Information Systems \\ Birkbeck, University of London \\ Malet Street, London WC1E 7HX \\ Email: gr@dcs.bbk.ac.uk
}

\begin{abstract}
Wireless sensor networks (WSN) are one of the enabling technologies for the Internet of Things. Due to the restricted capability of sensor, the performance of WSN is sensitive to interference from nearby wireless devices utilizing the same band. The channel hopping technique is adopted by IEEE 802.15.4e standard to mitigate such adverse effect. In this paper we propose the Adaptive Time-slotted Channel Hopping (A-TSCH) framework which augments standard IEEE 802.15.4e channel hopping with mechanisms for learning the spectral condition and blacklisting. Complete design and implementation are provided, followed by analysis of experimental results. We discover that A-TSCH improves the packet delivery in the presence of interference from co-located devices, leading to substantially enhanced reliability of WSN based on IEEE 802.15.4e.
\end{abstract}

\section{INTRODUCTION}

Wireless sensor networks (WSN) are one of the core ingredients of the Internet of Things. Current developments in wireless communication have led to intensified utilization of the $2.4 \mathrm{GHz}$ Industrial Scientific and Medical (ISM) band [1] where most WSN operate. This poses a challenge as sensors have to compete with a variety of other devices for the scarce spectrum. Channel hopping technique was developed to mitigate this issue [2], [3] by regularly switching communication frequencies in a pseudo-random manner [4]. This approach has been widely adopted [5], [6] and is now part of the IEEE 802.15.4e standard [7].

The standard channel hopping is limited since it does not consider differences between IEEE 802.15.4 channels. In this paper, we propose the Adaptive Time-slotted Channel Hopping (A-TSCH) framework which addresses such limitation of existing IEEE 802.15.4e channel hopping by only utilizing a selective set of desirable channels for hopping. The design of A-TSCH is presented in detail; implementation is carried out on a WSN platform and its performance is evaluated through experiments, highlighting the improvement of reliability as well as the validity of the A-TSCH framework.

The remainder of the paper is structured as follows: Section II reviews some studies related to this work. The overview of A-TSCH is provided in Section III and detailed descriptions of two vital components are presented subsequently in Section IV and V. The performance of A-TSCH is evaluated through experiments described in Section VI, and results are analyzed in Section VII to demonstrate our findings. Finally conclusions are drawn in Section VIII.

\section{RELATED WORK}

Using pseudo-randomized frequency access to allay adverse effect of ambient interference is an effective strategy embraced by several technologies operating in the ISM band, including Bluetooth and WirelessHART [5], [6], and a formal framework known as Time-slotted Channel Hopping (TSCH) is defined in IEEE 802.15.4e MAC Enhancement Standard [7]. TSCH divides time into discrete timeslots and uses pseudo-randomly chosen operating frequencies in individual timeslots [4], [8]. Accordingly, transmission is evenly spread over the entire set of 16 channels in long term [7].

The effectiveness of channel hopping essentially comes from the variation of desirability among available channels [9], [10]. While single-frequency schemes suffer significant degradation if its channel experiences powerful interference, channel hopping distributes the risk of negative influences over a wide spectral range, considerable enhancing the overall reliability [4], [11]. Taking this philosophy one step further, the blacklisting technique aims to use only "good" channels for hopping. Some supporting evidence for this approach is found in [11]. Specifically, communication traces between TSCH-based sensors were recorded and subsequently replayed with certain channels removed from the hopping sequence. An improvement in packet delivery ratio (PDR) was observed as a result. Although this finding was purely statistical and few suggestion on specific design was given, it motivated the idea of designing a blacklisting algorithm for $\mathrm{TSCH}$, which is an essential component of A-TSCH.

The Efficient Multichannel MAC (EM-MAC) protocol [12] demonstrates a practical design of blacklisting mechanism. In EM-MAC, certain parameters are regularly exchanged to enable senders to deduce the wake-up time and pseudorandomized communication frequencies of the receiving end. Right before the transmission of outgoing packets, clear channel assessments (CCA) is performed to detect whether the channel is interference-free. Channels are blacklisted if either CCA or packet delivery fails three times and senders are inform of updated blacklists by corresponding receivers. 


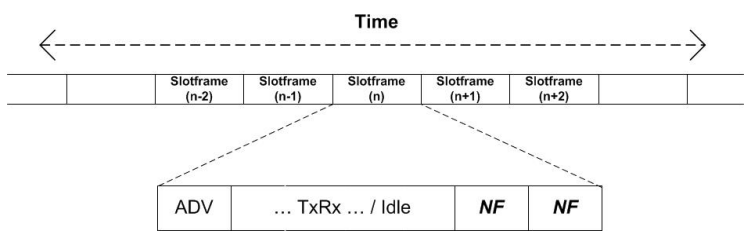

Fig. 1: Slotframes of standard TSCH include ADV, Tx, Rx and Idle type. A-TSCH introduces an additional NF type.

Although EM-MAC and A-TSCH share the same philosophy of employing blacklisting, there are several importan$\mathrm{t}$ differences. First, transmission in EM-MAC is receiverinitiated as senders only transmit after confirmed by receivers. In contrast, A-TSCH communications happen in scheduled timeslots and require no additional coordination once the network is established. Second, in EM-MAC only senders have the opportunity to probe channel desirability, whereas all node has such ability in A-TSCH. Third, blacklisting in EM-MAC is either invoked by failed CCA or unsuccessful packet delivery, which are a posteriori knowledge of affected performance. The $\mathrm{A}-\mathrm{TSCH}$, on the other hand, regularly monitors the spectral condition thus more proactively protects performance from potential adverse factors.

\section{Adaptive Time-Slotted Channel Hopping} (A-TSCH)

Adaptive Time-slotted Channel Hopping (A-TSCH) is a MAC layer framework for WSN. It extends the IEEE 802.15.4e Enhancement Standard [7] through augmenting the existing channel hopping mechanism. This section begins with a specification of standard IEEE 802.15.4e TSCH and then proceeds to provide an overview of the A-TSCH architecture.

\section{A. Standard IEEE 802.15.4e TSCH}

IEEE 802.15.4e TSCH [7] divides time into successive timeslots. Repetitive patterns of timeslots are grouped into slotframes. Therefore the temporal domain is considered an infinite series of slotframes consisting of fixed timeslot arrangement, as illustrated in Figure 1. Activities inside timeslots depend on associated type values. The standard TSCH has 3 types of non-idle slots: advertisement (ADV), transmission (Tx) and reception (Rx). Synchronized nodes maintain a uniform sense of time by sharing identical counts of elapsed timeslots, known as Absolute Slot Number (ASN). Accordingly, slotframes are aligned throughout the network which enables coordinated operations.

$$
\text { Channel }=(\text { ASN } \bmod 16)+11
$$

Operating frequencies in TSCH are pseudo-randomly generated using Equation (1). For $\mathrm{ASN} \in[0,+\infty)$ the equation yields Channel $\in[11,26]$, hence providing indiscriminate pseudo-random access to all sixteen IEEE 802.15.4 channels in $2.4 \mathrm{GHz}$ band.

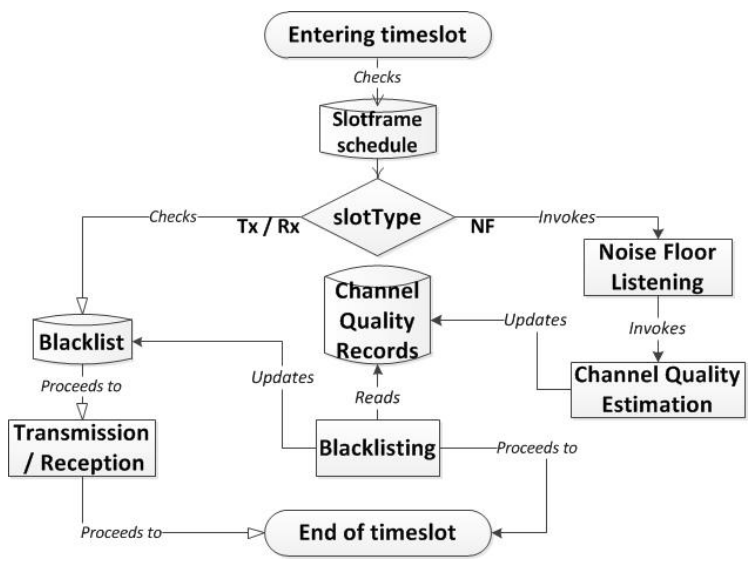

Fig. 2: A-TSCH components and operations. Arrow types correspond to logic branches. Lines are annotated with actions.

\section{B. A-TSCH Design}

To facilitate blacklisting in A-TSCH, the standard TSCH slotframe is modified to include two additional Noise Floor (NF) slots that are reserved for noise floor listening, as illustrated in Figure 1. Transmission is suspended in NF slots to ensure sampled noise floors are not affected by network's own signals [13]. This particular placement provides fairly frequent opportunities for noise detection without incurring over-sized slotframe which may affect network throughput. In practice, noise floors are retrieved from the Received Signal Strength Indicator (RSSI) register of the radio [14]. The hardware also report another Link Quality Indicator (LQI) value, but its correlation with transmission success rate is not as good as RSSI's [15], [16], especially for unstable transmission medium [17].

The logic flow of A-TSCH is illustrated in Figure 2. Upon entering a timeslot, A-TSCH firstly discovers the slot type by checking the schedule. In NF timeslots, the noise floor listening process is invoked. Equation (1) is used to indiscriminately and pseudo-randomly determine channels to probe. Collected noise readings are passed to the channel quality estimation which updates channel quality records to reflect the desirability of channels. Blacklisting subsequently composes the blacklist which is the collection of undesirable channels to be excluded from the hopping sequence. In Tx or Rx slots, normal communication routines are carried out using frequencies generated by Equation (1) while consulting the blacklist. The processes of channel quality estimator and blacklisting are vital ingredients of A-TSCH and for this reason they are further discussed in the following sections.

\section{Channel Quality Estimator}

The obtained noise floor readings represent momentary interference levels and may not reflect channel quality over longer periods. Accordingly, exponential smoothing (ES) technique is adopted to provide a simple and lightweight channel quality estimator for low power wireless sensors. After a brief introduction to properties of ES, its operating parameter is 
TABLE I: Smoothing coefficient candidate with corresponding forecast accuracy

\begin{tabular}{|l|c|c|c|c|c|c|c|c|c|}
\hline $\mathbf{0 . 1}$ & $\mathbf{0 . 2}$ & $\mathbf{0 . 3}$ & $\mathbf{0 . 4}$ & $\mathbf{0 . 5}$ & $\mathbf{0 . 6}$ & $\mathbf{0 . 7}$ & $\mathbf{0 . 8}$ & $\mathbf{0 . 9}$ & $\mathbf{1 . 0}$ \\
\hline 2.47 & 2.50 & 2.52 & 2.54 & 2.56 & 2.57 & 2.57 & 2.59 & 2.61 & 2.88 \\
\hline
\end{tabular}

tuned through simulation. An additional ES-based estimator that utilizes Kalman Filter for dynamic parameter adjustment is also proposed.

\section{A. Exponential Smoothing}

Exponential smoothing (ES) is a popular forecast technique known for its effectiveness and relative simplicity [18], [19] and it has several variants for estimation subject with various characteristics. Single exponential smoothing, also known as simple exponential smoothing, is arguably the most concise form of ES techniques. Due to its independence from assumptions of underlying process and low computational overhead, it is suitable to be used in power-constrained wireless sensors.

$$
\hat{Y}_{t+1 \mid t}=\alpha O_{t}+(1-\alpha) Y_{t \mid t-1}
$$

Equation (2) generalizes the smoothing process. $O_{t}$ is the noise observed at time $t$ and $Y_{t \mid t-1}$ denotes the forecast for time $t$ previously generated at $t-1$. And $\hat{Y}_{t+1 \mid t}$, the forecast for time $t+1$ yielded at $t$ is the weighted combination of $O_{t}$ and $Y_{t \mid t-1}$, subject to a smoothing coefficient $\alpha \in[0,1]$. Accordingly, a forecast with single ES is yielded entirely from the result of previous steps and most recent observation, ensuring modest computation and storage overheads.

It is evident that the estimator is controlled by coefficient $\alpha$. This parameter is case-specific and simulations on real noise samples were conducted to determine suitable $\alpha$ for our experiment environment. Noise were sampled using Guidance and Inertial Navigation Assistant (Gina) motes [20] for a total duration of 48 hours. Candidate coefficients were evenly extracted between 0.1 to 1.0 with a step of 0.1 . Zero was not considered because it would nullify the influence of observations in Equation (2) which effectively disables noise floor listening. Simulations were conducted in Matlab and the root-mean-square error (RMSE) between the true values and forecasts are shown in Table I. The $\alpha$ of 0.1 was found to yield the best result thus later used in experiments.

\section{B. Exponential Smoothing aided by Kalman Filter (KFES)}

An additional type of estimator is devised in this work to dynamically adjust $\alpha$ by employing Kalman Filter (KF), an optimal estimator for dynamic linear systems, with the assumption that the best smoothing coefficient tends to be constant in long term. This estimator is called KFES and described in Algorithm 1. Note that details of ES and KF functions are omitted from the algorithm listing since they are well known and also due to the page limit. Specifically, noise samples are thought to truthfully reflect momentary states.
Thus it is possible to determine the $\alpha$ which could have led to optimal estimates in hindsight. This retrospective information is denoted as bestAlpha and used as the correction value for $\mathrm{KF}$. The estimate generated by $\mathrm{KF}$ is taken as the ideal $\alpha$ and subsequently used by ES to yield noise forecasts.

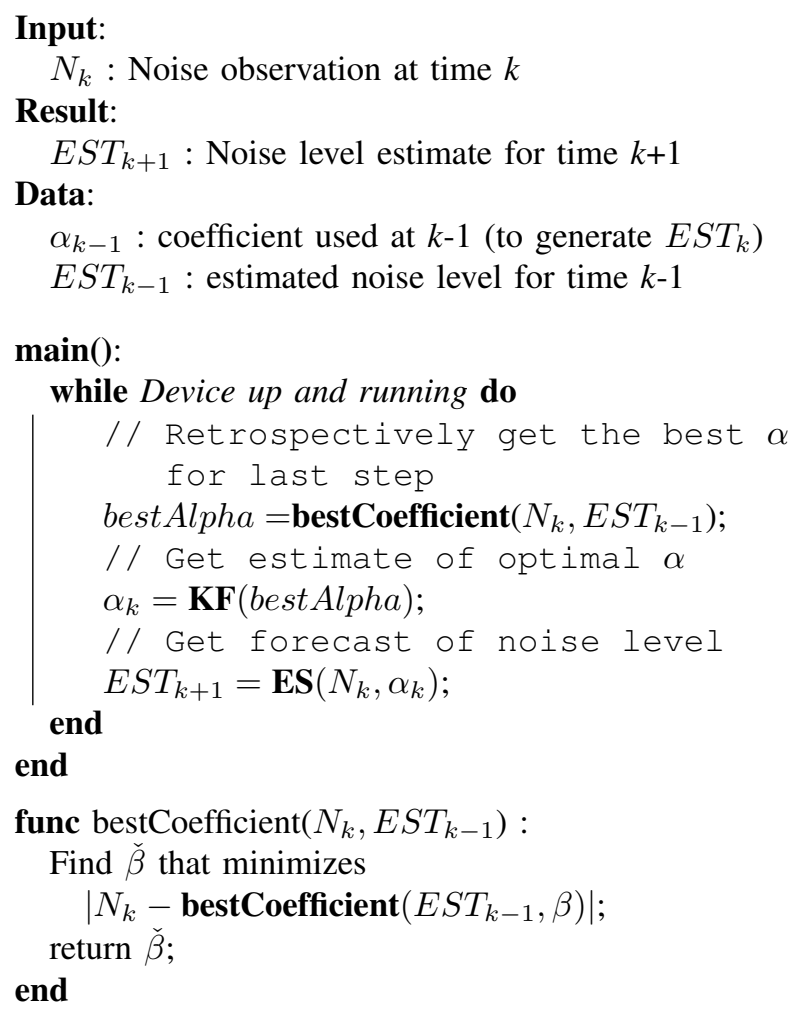

Algorithm 1: The algorithm of KFES estimator

\section{BLACKLISTING}

A blacklist consists of 16 bits that represent individual frequencies and channels are blacklisted by setting corresponding bits to 1 . The sizing of blacklist controls the maximum number of channels allowed to excluded. This parameter can be either static or, alternatively, dynamic to cover any channel falling short of certain threshold, provided that at least one channel remains usable.

In Tx / Rx slots, blacklists are consulted when nodes initiate access to radio frequencies obtained from Equation (1). The communication goes ahead if the prospective channel is not blacklisted; otherwise an alternative must be generated with Equation (1) and checked again. This iterative process terminates when an admissible channel is found.

Blacklists are periodically updated at intervals of $T_{u}$ to reflect latest spectral condition. The synchronization of blacklists is crucial to maintaining communication between peers. In ADV slots, nodes insert their local blacklists to ADV payload and propagation is achieved simultaneously with standard TSCH timing synchronization [14]. An 
important detail is that blacklisting is deactivated in ADV slots so that common hopping sequence can be easily recovered in case of desynchronized blacklists since ADV packets are always exchanged using default hopping sequence.

\section{IMPLEMENTATION AND EXPERIMENT}

In order to test the performance of the proposed framework, A-TSCH was implemented atop Berkeley's OpenWSN [21] protocol stack. Experiments were conducted in a communal workspace environment (approximately $12 m \times 12 m$ ) with 3 wireless access points placed in proximity and typically more than 10 WLANs visible. A total of 6 Guidance and Inertial Navigation Assistant (Gina) nodes [20] were deployed as 3 sender-receiver pairs. Note that A-TSCH does not depend on specific hardware. One of the groups was configured with unmodified TSCH while the other two adopted A-TSCH with ES and KFES estimators, respectively. Senders were programmed to simultaneously transmit 600 packets to their corresponding partners at power of $0 \mathrm{dBm}$ with inter-packet time of 2 seconds. To help demonstrate the effect of blacklisting, the update interval $T_{u}$ was set to 200 seconds to ensure at least 5 update opportunities within the duration. Experiments were conducted in 4 rounds using blacklist sizes of 3, 6, 9 and 12, respectively, and each round was repeated 6 times.

\section{ANALYSIS}

The metric of Expected Transmission Count (ETX) is empolyed to quantify the transmission success rate. Since ETX is the reciprocal of packet delivery ratio (PDR), therefore it denotes the average number of transmission required for a packet to be successfully received [11], [22].

The ETX observed in experiments are reported in Figure 3. The individual segments denote results achieved with different blacklist sizes as labeled on the horizontal axis, while bars inside each segment represent ETX performance with different estimators. In particular, the standard TSCH has estimator type of N/A as it does not use any, and its ETX results are used in all segments as the baseline for measuring the effect of A-TSCH. It can be clearly observed in the figure that motes using A-TSCH with any settings uniformly deliver lower ETXs than standard TSCH, confirming that A-TSCH improves transmission success rate. Reduction in ETX is quantified in percentage and labeled atop of corresponding bars in Figure 3.

Relationship between ETX and blacklist size is evident as size 12 delivers ETX reduction nearly twice as much as size 3 does, while size 6 and 9 provide similar results. Variation in ETX reduction also exists between different estimator types. While the KFES outperforms its ES-based counterpart when small or large blacklist (size 3 and 12) are used, ES exhibits marginal advantage with moderate sizes (6 and 9). Although these findings are not sufficient to derive any deterministic arguments about the effect of A-TSCH parameters, they

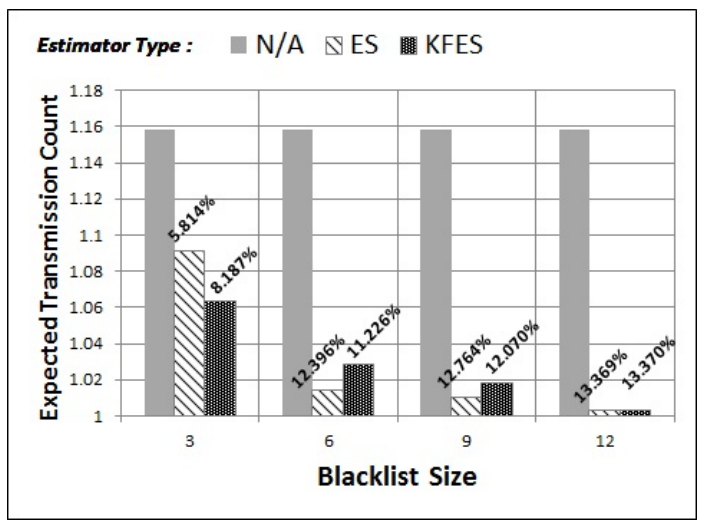

Fig. 3: ETX obtained with different blacklist sizes and estimators. ETX reduction is labeled in percentage.

provide clues about what future work should investigate.

The blacklist updates results are also examined to ascertain whether A-TSCH was able to correctly blacklist channels in accord with noise floor condition. To this end, the auxiliary metric of Blacklisting Rate (BR) is introduced and defined as the number of times that certain channel is blacklisted divided by the number of blacklist updates. Accordingly, BR denotes how frequently a channel is recognized as noisy and excluded by the blacklisting process. The actual channel condition was monitored using Wi-Spy spectrum analyzer [23] and the obtained noise density is presented in Figure 4(a) as a baseline.

Channel BR associated with different blacklist sizes are illustrated in Figure 4(b) and found to be in agreement with the actual noise condition. Despite occasional variations, the most noisy channels (11 through 14 and 16 through 19, according to Figure 4(a)), were very frequently blacklisted; the relatively quiet channel 25 and 26 were largely intact. This also partly explains the diversity of ETX among blacklist sizes: the size of 12 can exclude nearly all noisy channels (11 through 19, 22 and 23), hence the biggest reduction; as the blacklist sizes decrease, some noisy channels inevitably escaped the exclusion and exerted negative influences.

Also plotted is the BR's association with estimators in Figure 4(c). It is observed that distinction between channel BRs is sharper with ES than KFES. A possible reason is that the ES estimator was less sensitive to momentary fluctuations in noise level because the low smoothing factor (0.1) made its view of channel desirability relatively steady. In comparison, KFES was more susceptible to short-term changes in noise levels hence the less deterministic BR profile. These explanations are in accord with the fact that KFES performed best with small blacklists (4) and ES tended to excel with bigger ones: blacklists large enough to contain all noisy channels could benefit from the stability of ES estimator which led to more desirable results; and in case of small blacklists, KFES's actively adjusted evaluation allowed for accurate identification of the most noisy frequencies. 


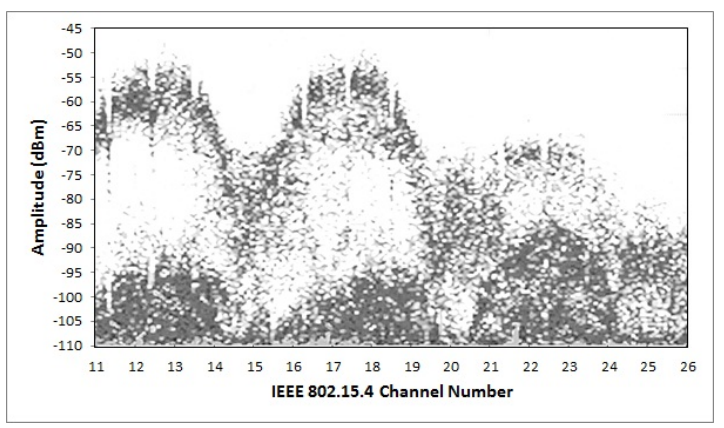

(a)

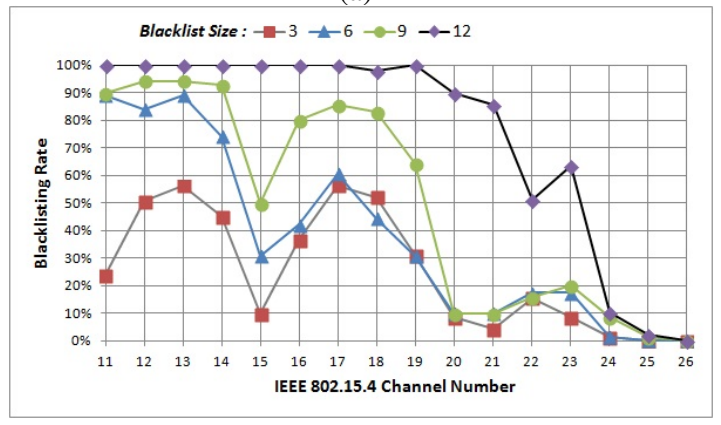

(b)

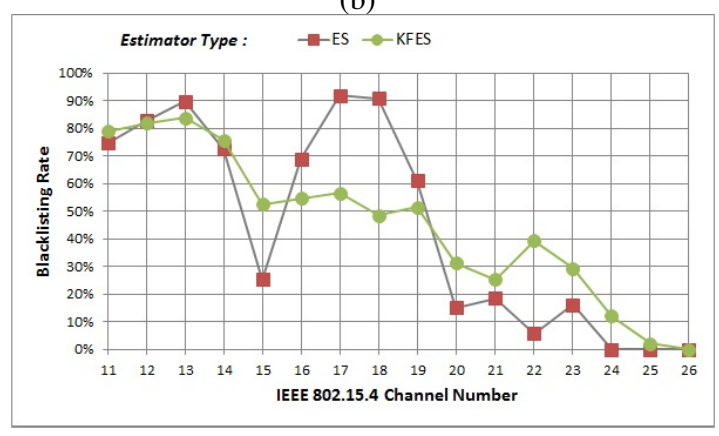

(c)

Fig. 4: (a) WiSpy's noise density graph; Blacklisting rates (BR) associated with (b) blacklist sizes and (c) estimators.

In spite of the diversity discussed above, all BR curves of BR conform to the noise density in 4(a), confirming the overall correctness of the A-TSCH blacklisting process. However, the above findings may not be independent from specific environments, therefore investigations should be furthered in the continuation of this work, for example, into an adaptive blacklist sizing mechanism.

\section{CONCLUSION}

In this paper, we briefly reviewed the IEEE 802.15.4e Timeslotted Channel Hopping (TSCH) and suggested further improvement. The Adaptive TSCH (A-TSCH) was subsequently proposed to improve the reliability of transmission in the face of interference via blacklisting technique which exclude channels of high noise levels from hopping sequence. The A-TSCH was implemented for a WSN platform and tests were conducted with different estimators and blacklist sizes. A commercial spectrum analyzer was employed to verify the correctness of blacklisting operations. Although no singular best estimator technique and blacklist configuration could be identified based on current findings, the experimental evidences clearly demonstrate A-TSCH's advantage over standard IEEE 802.15.4e channel hopping in terms of transmission success rate in the face of interference.

\section{REFERENCES}

[1] S. Pollin et al., "Distributed cognitive coexistence of 802.15 .4 with 802.11," in 1st Int. Conf. Cognitive Radio Oriented Wireless Networks and Comm., 2006.

[2] H. Khaleel et al., "Impact of Wi-Fi traffic on the IEEE 802.15.4 channels occupation in indoor environments," in Int. Conf. Electromagnetics in Advanced Applications, 2009. ICEAA '09., 2009, pp. 1042 - 1045.

[3] C. M. D. Dominicis et al., "Investigating WirelessHART coexistence issues through a specifically designed simulator," in Instrumentation and Measurement Technol. Conf. 2009 IEEE. IEEE, 2009, pp. 1085-1090.

[4] K. S. J. Pister and L. Doherty, "TSMP: Time Synchronized Mesh Protocol," in Parallel and Distributed Computing Systems, 2008.

[5] Bluetooth Core Specifications version 4.0, SIG Bluetooth Std.

[6] "WirelessHART technical data sheet."

[7] "IEEE standard for local and metropolitan area networks-part 15.4: Low-rate wireless personal area networks (lr-wpans) amendment 1: Mac sublayer," IEEE Std 802.15.4e-2012 (Amendment to IEEE Std 802.15.42011), pp. $1-225,162012$.

[8] A. Tinka et al., "A decentralized scheduling algorithm for time synchronized channel hopping," ICST Transactions on Mobile Communications and Applications, vol. 11, no. 7-9, September 2011.

[9] B. Kerkez et al., "Feasibility analysis of controller design for adaptive channel hopping," in Proc. 4th VALUETOOLS. ICST, 2009, pp. 76:176:6.

[10] H. Khaleel et al., "Distributed spectrum sensing and channel selection in opportunistic wireless personal area networks," in Proc. 2nd Int. Workshop on Mobile Opportunistic Networking, ser. MobiOpp '10, 2010, pp. $185-187$.

[11] T. Watteyne, A. Mehta, and K. Pister, "Reliability through frequency diversity: why channel hopping makes sense," in Proc. 6th ACM PEWASUN. ACM, 2009, pp. 116-123.

[12] L. Tang et al., "Em-mac: a dynamic multichannel energy-efficient mac protocol for wireless sensor networks," in Proceedings of the Twelfth ACM International Symposium on Mobile Ad Hoc Networking and Computing, ser. MobiHoc '11. New York, NY, USA: ACM, 2011, pp. 23:1-23:11.

[13] T. Yucek and H. Arslan, "A survey of spectrum sensing algorithms for cognitive radio applications," IEEE Commun. Surveys Tuts., vol. 11, pp. $116-130,2009$.

[14] P. Du and G. Roussos, "Adaptive channel hopping for wireless sensor networks," in 2011 International Conference on Selected Topics in Mobile and Wireless Networking (iCOST), 2011.

[15] K. Srinivasan and P. Levis, "RSSI is under appreciated," in Proceedings of the Third Workshop on Embedded Networked Sensors, 2006.

[16] K. Benkic et al., "Using RSSI value for distance estimation in wireless sensor networks based on ZigBee," in Systems, Signals and Image Processing, 2008. 15th International Conference on, June, pp. 303-306.

[17] M. Rondinone et al., "Designing a reliable and stable link quality metric for wireless sensor networks," in Proceedings of the workshop on Realworld wireless sensor networks, ser. REALWSN '08, 2008, pp. 6-10.

[18] E. S. Gardner and D. G. Dannenbring, "Forecasting with exponential smoothing: Some guidelines for model selection," Decision Sciences, vol. 11, no. 2, pp. 370-383, 1980.

[19] O. Y. Ozalt, B. Hunsaker, and A. J. Schaefer, "Predicting the solution time of branch-and-bound algorithms for mixed-integer programs," INFORMS J. on Computing, vol. 23, no. 3, pp. 392-403, Jul. 2011.

[20] "Warpwing project page." [Online]. Available: http://warpwing. sourceforge.net/

[21] Berkeley's openwsn. [Online]. Available: https://openwsn.atlassian.net/ wiki/display/OW

[22] D. S. J. D. Couto, "High-throughput routing for multi-hop wireless networks," Ph.D. dissertation, MIT, June 2004.

[23] Wi-spy channel analyzer. Wi-Spy. [Online]. Available: http://www. metageek.net/products/wi-spy/ 